\title{
Friction factors for smooth pipe flow
}

\author{
By B. J. MCKEON ${ }^{1} \dagger$, C. J. SWANSON $\mathrm{SW}^{2}$, M. V. ZA GAR O LA $\mathrm{A}^{1}$, \\ R. J. DONNELL Y ${ }^{2}$ AND A. J. SMIT S $^{1}$ \\ ${ }^{1}$ Department of Mechanical and Aerospace Engineering, Princeton University \\ Princeton, NJ 08544-0710, USA \\ ${ }^{2}$ Department of Physics, University of Oregon, Eugene, OR 97403-1274, USA
}

(Received 27 April 2004 and in revised form 28 April 2004)

Friction factor data from two recent pipe flow experiments are combined to provide a comprehensive picture of the friction factor variation for Reynolds numbers from 10 to $36,000,000$.

For pipe flow, as long as entrance effects, roughness, and temperature variations are small, dimensional analysis indicates that the friction factor $\lambda$ is only a function of the Reynolds number $R e$. That is,

$$
\lambda=f(R e)
$$

where

$$
\lambda=\frac{-(\mathrm{d} P / \mathrm{d} x) D}{\frac{1}{2} \rho \bar{U}^{2}} \quad \text { and } \quad R e=\frac{\bar{U} D}{v} .
$$

Here $f$ denotes a functional relationship, $\mathrm{d} P / \mathrm{d} x$ is the pressure drop per unit length, $D$ is the diameter of the pipe, $\rho$ is the fluid density, $v$ is the kinematic viscosity, and $\bar{U}$ is the flow velocity averaged over the cross-sectional area of the pipe.

The Princeton (Zagarola \& Smits 1998; McKeon et al. 2004a; McKeon, Zagarola \& Smits 2004b) and Oregon (Swanson et al. 2002) research groups have recently examined fully developed pipe flow using very different apparatus. Compressed air is used in the Princeton 'Superpipe', whereas the Oregon device uses several roomtemperature gases: helium, oxygen, nitrogen, carbon dioxide and sulphur hexafluoride are used for relatively small Reynolds numbers, and normal liquid helium (helium I) is used for the highest Reynolds numbers. The difference in scale of the two devices is dramatic: for example, the Superpipe weighs about 25 tons, whereas the Oregon tube weighs about 1 ounce.

The combined data sets are tabulated in table 1 and plotted in figures 1 and 2 . We see that the range of experimentally known friction factors has been increased from $R e=10^{3}$ to $R e=3.2 \times 10^{6}$ (see, for example, figure 20.1 in Schlichting 1979), and $R e=10$ to $R e=35.7 \times 10^{6}$. Where they overlap, the data lie within an average of $1.7 \%$, compared with experimental errors of $2 \%$ to $4 \%$ for the Oregon data and

$\dagger$ Current address: Department of Aeronautics, Imperial College, London SW7 2BY, UK. $\ddagger$ Current address: Gutenberg College, 1883 University St., Eugene, OR 97401, USA.

I Current address: Creare Inc, Etna Road Box 71 Hanover, NH 03755, USA. 


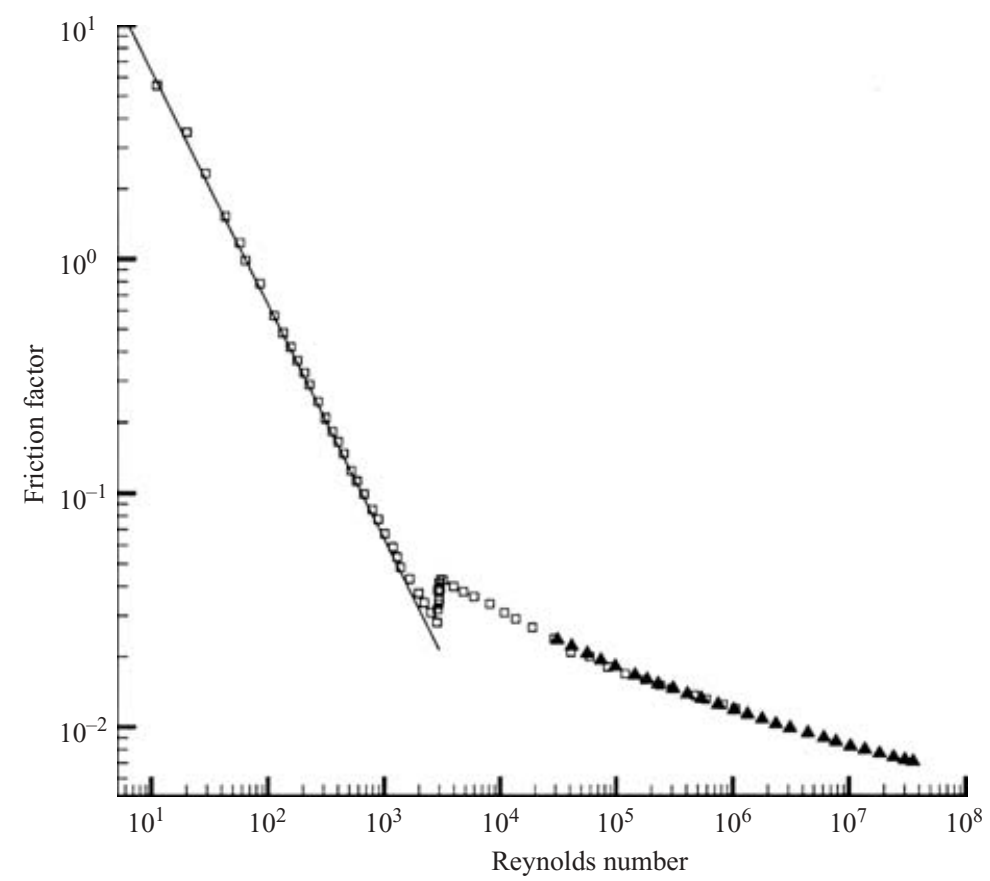

FIgURE 1. Combined data sets from table 1: $\boldsymbol{\Lambda}$, Princeton; $\square$, Oregon; - , theoretical friction factor for laminar flow, $\lambda=64 / R e$. Since the error bars are smaller than the size of the symbols, they are not shown.

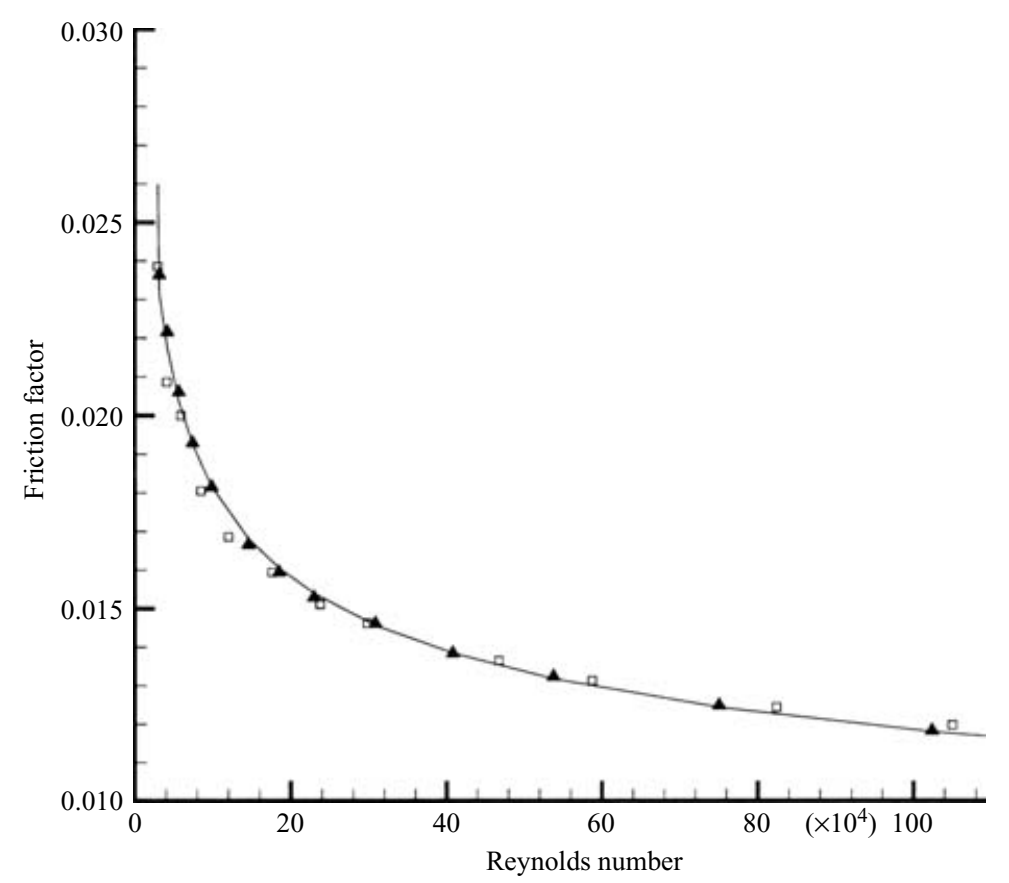

Figure 2. Linear plot of the points where the two data sets overlap. $\boldsymbol{\Lambda}$, Princeton; $\square$, Oregon. The line corresponds to equation (1). 


\begin{tabular}{|c|c|c|c|c|c|}
\hline \multicolumn{4}{|c|}{ Oregon data } & \multicolumn{2}{|c|}{ Princeton data } \\
\hline$R e$ & $\lambda$ & $R e$ & $\lambda$ & $R e$ & $\lambda$ \\
\hline $1.121 \mathrm{E}+01$ & $5.537 \mathrm{E}+00$ & $2.227 \mathrm{E}+03$ & $3.405 \mathrm{E}-02$ & $3.131 \mathrm{E}+04$ & $2.364 \mathrm{E}-02$ \\
\hline $2.022 \mathrm{E}+01$ & $3.492 \mathrm{E}+00$ & $2.554 \mathrm{E}+03$ & $3.091 \mathrm{E}-02$ & $4.144 \mathrm{E}+04$ & $2.216 \mathrm{E}-02$ \\
\hline $2.928 \mathrm{E}+01$ & $2.329 \mathrm{E}+00$ & $2.868 \mathrm{E}+03$ & $2.804 \mathrm{E}-02$ & $5.636 \mathrm{E}+04$ & $2.061 \mathrm{E}-02$ \\
\hline $4.319 \mathrm{E}+01$ & $1.523 \mathrm{E}+00$ & $2.903 E+03$ & $3.182 \mathrm{E}-02$ & $7.397 \mathrm{E}+04$ & $1.929 \mathrm{E}-02$ \\
\hline $5.773 \mathrm{E}+01$ & $1.173 \mathrm{E}+00$ & $2.926 \mathrm{E}+03$ & $3.846 \mathrm{E}-02$ & $9.846 \mathrm{E}+04$ & $1.815 \mathrm{E}-02$ \\
\hline $6.458 \mathrm{E}+01$ & $9.863 \mathrm{E}-01$ & $2.955 E+03$ & $3.363 \mathrm{E}-02$ & $1.456 \mathrm{E}+05$ & $1.666 \mathrm{E}-02$ \\
\hline $8.605 E+01$ & $7.826 \mathrm{E}-01$ & $2.991 \mathrm{E}+03$ & 4.124E-02 & $1.848 \mathrm{E}+05$ & $1.594 \mathrm{E}-02$ \\
\hline $1.133 \mathrm{E}+02$ & $5.709 \mathrm{E}-01$ & $2.997 \mathrm{E}+03$ & $3.500 \mathrm{E}-02$ & $2.296 \mathrm{E}+05$ & $1.529 \mathrm{E}-02$ \\
\hline $1.353 \mathrm{E}+02$ & $4.815 \mathrm{E}-01$ & $3.047 \mathrm{E}+03$ & $3.875 \mathrm{E}-02$ & $3.085 \mathrm{E}+05$ & $1.461 \mathrm{E}-02$ \\
\hline $1.575 \mathrm{E}+02$ & 4.182E-01 & $3.080 \mathrm{E}+03$ & $4.285 \mathrm{E}-02$ & $4.081 \mathrm{E}+05$ & $1.384 \mathrm{E}-02$ \\
\hline $1.794 \mathrm{E}+02$ & $3.655 \mathrm{E}-01$ & $3.264 \mathrm{E}+03$ & $4.260 \mathrm{E}-02$ & $5.378 \mathrm{E}+05$ & $1.324 \mathrm{E}-02$ \\
\hline $2.064 \mathrm{E}+02$ & $3.237 \mathrm{E}-01$ & $3.980 \mathrm{E}+03$ & $3.995 \mathrm{E}-02$ & $7.507 \mathrm{E}+05$ & $1.249 \mathrm{E}-02$ \\
\hline $2.280 \mathrm{E}+02$ & $2.884 \mathrm{E}-01$ & $4.835 E+03$ & $3.797 \mathrm{E}-02$ & $1.024 \mathrm{E}+06$ & $1.183 \mathrm{E}-02$ \\
\hline $2.709 \mathrm{E}+02$ & $2.433 \mathrm{E}-01$ & $5.959 \mathrm{E}+03$ & $3.610 \mathrm{E}-02$ & $1.342 \mathrm{E}+06$ & $1.131 \mathrm{E}-02$ \\
\hline $3.152 \mathrm{E}+02$ & $2.077 \mathrm{E}-01$ & $8.162 \mathrm{E}+03$ & $3.364 \mathrm{E}-02$ & $1.791 \mathrm{E}+06$ & $1.079 \mathrm{E}-02$ \\
\hline $3.589 \mathrm{E}+02$ & $1.834 \mathrm{E}-01$ & $1.090 \mathrm{E}+04$ & $3.088 \mathrm{E}-02$ & $2.352 \mathrm{E}+06$ & $1.028 \mathrm{E}-02$ \\
\hline $4.029 \mathrm{E}+02$ & $1.656 \mathrm{E}-01$ & $1.365 \mathrm{E}+04$ & 2.903E-02 & $3.109 \mathrm{E}+06$ & $9.890 \mathrm{E}-03$ \\
\hline $4.502 \mathrm{E}+02$ & $1.475 \mathrm{E}-01$ & $1.899 \mathrm{E}+04$ & $2.670 \mathrm{E}-02$ & $4.438 \mathrm{E}+06$ & $9.410 \mathrm{E}-03$ \\
\hline $5.225 \mathrm{E}+02$ & $1.245 \mathrm{E}-01$ & $2.943 E+04$ & $2.386 \mathrm{E}-02$ & $6.103 E+06$ & $8.970 \mathrm{E}-03$ \\
\hline $5.831 \mathrm{E}+02$ & $1.126 \mathrm{E}-01$ & $4.085 E+04$ & $2.086 \mathrm{E}-02$ & $7.757 \mathrm{E}+06$ & $8.620 \mathrm{E}-03$ \\
\hline $6.718 \mathrm{E}+02$ & $9.917 \mathrm{E}-02$ & $5.922 \mathrm{E}+04$ & $2.000 \mathrm{E}-02$ & $1.031 \mathrm{E}+07$ & $8.250 \mathrm{E}-03$ \\
\hline $7.898 \mathrm{E}+02$ & $8.501 \mathrm{E}-02$ & $8.476 \mathrm{E}+04$ & $1.805 \mathrm{E}-02$ & $1.368 \mathrm{E}+07$ & $7.980 \mathrm{E}-03$ \\
\hline $8.910 \mathrm{E}+02$ & $7.722 \mathrm{E}-02$ & $1.200 \mathrm{E}+05$ & $1.686 \mathrm{E}-02$ & $1.830 \mathrm{E}+07$ & $7.670 \mathrm{E}-03$ \\
\hline $1.013 \mathrm{E}+03$ & $6.707 \mathrm{E}-02$ & $1.760 \mathrm{E}+05$ & $1.594 \mathrm{E}-02$ & $2.413 \mathrm{E}+07$ & $7.400 \mathrm{E}-03$ \\
\hline $1.197 \mathrm{E}+03$ & $5.880 \mathrm{E}-02$ & $2.377 \mathrm{E}+05$ & $1.511 \mathrm{E}-02$ & $3.015 \mathrm{E}+07$ & $7.200 \mathrm{E}-03$ \\
\hline $1.300 \mathrm{E}+03$ & $5.328 \mathrm{E}-02$ & $2.982 \mathrm{E}+05$ & $1.462 \mathrm{E}-02$ & $3.554 \mathrm{E}+07$ & $7.080 \mathrm{E}-03$ \\
\hline $1.390 \mathrm{E}+03$ & $4.815 \mathrm{E}-02$ & $4.678 \mathrm{E}+05$ & $1.365 \mathrm{E}-02$ & & \\
\hline & 4.304E-02 & $5.875 \mathrm{E}+05$ & $1.313 \mathrm{E}-02$ & & \\
\hline \multirow[t]{2}{*}{$1.994 \mathrm{E}+03$} & $3.739 \mathrm{E}-02$ & $8.242 \mathrm{E}+05$ & $1.244 \mathrm{E}-02$ & & \\
\hline & & $1.050 \mathrm{E}+06$ & $1.198 \mathrm{E}-02$ & & \\
\hline
\end{tabular}

TABLE 1. Friction factor data from the two experiments.

$1.1 \%$ for the Princeton data. The line shown in figure 2 is the curve fit proposed by McKeon et al. (2004b) for $\operatorname{Re}_{D}>300 \times 10^{3}$ :

$$
\frac{1}{\lambda^{1 / 2}}=1.930 \log \left(\operatorname{Re} \lambda^{1 / 2}\right)-0.537 .
$$

The maximum difference between equation (1) and the Princeton data for $31 \times 10^{3} \leqslant$ $R e \leqslant 35 \times 10^{6}$ is always less than $1.25 \%$. For $300 \times 10^{3} \leqslant R e \leqslant 13.6 \times 10^{6}$, the deviation from the proposed formula is less than $\pm 0.5 \%$. For Reynolds numbers above $13.6 \times 10^{6}$ the friction factors start to move above this line, indicating the possible effects of roughness, but the trend is within the experimental error on $\lambda$ for all but the highest Reynolds number.

Research at Princeton is supported by ONR under Grant N00014-03-1-0320, and by NSF under Grants No. CTS-9908442 and CTS-0306691. Research at Oregon is supported by the National Science Foundation under grant DMR 0202554. 
44 B. J. McKeon, C. J. Swanson, M. V. Zagarola, R. J. Donnelly and A. J. Smits

\section{REFERENCES}

McKeon, B. J., Li, J., Jiang, W., Morrison, J. F. \& Smits, A. J. $2004 a$ Further observations on the mean velocity distribution in fully-developed turbulent pipe flow. J. Fluid Mech. 501, 135-147.

McKeon, B. J., Zagarola, M. V. \& Smits, A. J. $2004 b$ A new friction factor relationship for turbulent pipe flow. J. Fluid Mech. (submitted).

Schlichting, H. 1979 Boundary-Layer Theory, 7th Edn. McGraw-Hill.

Swanson, C. J., Julian, B., Ihas, G. G. \& Donnelly, R. J. 2002 Pipe flow measurements over a wide range of Reynolds numbers using liquid helium and various gases. J. Fluid Mech. 461, $51-60$.

Zagarola, M. V. \& Smits, A. J. 1998 Mean flow scaling of turbulent pipe flow. J. Fluid. Mech. 373, $33-79$. 